\title{
Variations of Cervical Vertebrae after Expression of a Hox-1.1 Transgene in Mice
}

\author{
Michael Kessel, Rudi Balling, and Peter Gruss \\ Department of Molecular Cell Biology \\ Max Planck Institute of Biophysical Chemistry \\ 3400 Göttingen \\ Federal Republic of Germany
}

\section{Summary}

To understand the function of murine homeobox genes, a genetic analysis is mandatory. We generated gain-of-function mutants by introducing genomic sequences of the Hox-1.1 gene under the control of a chicken $\beta$-actin promoter into mice. Our previous data had shown that these transgenic mice are nonviable after birth and are born with craniofacial abnormalities. In a subsequent detailed analysis of severely affected animals, malformations of the basioccipital bone, the atlas, and the axis were observed. Manifestation of an additional vertebra, a proatlas, occurred at the craniocervical transition. The dominant interference of the Hox-1.1 transgene with developmental programs seems to occur around day 9 of gestation, the time of neural crest migration and somite differentiation. We discuss the resulting phenotype with respect to a developmental control function of Hox-1.1.

\section{Introduction}

Many developmental variations and mutations have been described for the fruit fly Drosophila melanogaster (Ouweneel, 1976; Nüsslein-Volhard and Wieschaus, 1980). They were the basis for cloning corresponding genes, among them a defined class designated as homeotic genes (Gehring and Hiromi, 1986; Scott et al., 1989). These genes are involved in the specification of positional information along the anteroposterior axis (Akam et al., 1988). A common element of these genes, the homeobox, is also found in vertebrate genes (McGinnis et al., 1984). At present, the function of vertebrate homeobox genes can only be deduced from circumstantial evidence such as developmentally and spatially regulated expression during embryogenesis. No causal relationship between a murine homeobox gene and any of the numerous spontaneous or induced mouse mutations has been established, although some candidate genes map near the Hox loci and have suggestive expression patterns. Thus, hypodactyly maps close to the Hox-1 cluster on chromosome 6, hammer-toe maps very close to the Hox-7.1 gene on chromosome 5 , and rachitherata maps close to the Hox- 5 cluster on chromosome 2 (Mock et al., 1987; Robert et al., 1989; Hill et al., 1989; Featherstone et al., 1988). However, the phenotype of these mutants is not apparently homeotic: hypodactyly and hammer-toe both affect limb development, causing either too few digits or flexed digits with webbed connecting tissue, and rachitherata mice exhibit fusions between the second and third cervical vertebrae and a rib vertebrae syndrome in the thoracolumbar region (Theiler, 1988). Notably, certain types of variations of the vertebral column have been previously discussed as examples for homeosis during the the original definition of this term by Bateson (1894) and also by Grüneberg (1952).

Vertebrates are segmented early in embryogenesis by the formation of somites, mesodermal condensations lateral to the neural tube. Sclerotomal cells from the caudal half of one somite join with cells from the cranial half of a following somite to form a prevertebra, which eventually develops into a vertebra (Baur, 1969; Dawes, 1930; Stern and Keynes, 1986; Verbout, 1985; for a schematic representation, see Figure 1). Only the most anterior somites, the five occipital somites, do not generate vertebrae but fuse to form the occipital bones surrounding the foramen magnum, the exit of the spinal cord into the vertebral column (Hayek, 1927; Reiter, 1944).

In predecessors of mammals the caudal half of the fifth occipital somite and the cranial half of the first cervical somite form a vertebra-like structure termed the proatlas (Hayek, 1922). This proatlas has been detected in fossil bones of dinosaurs, and rudiments are present in reptiles (Hayek, 1924; Jenkins, 1969). In mammals, however, the first of the seven cervical vertebrae is the atlas, which is the only vertebra still possessing a hypochordal bar (anterior arch of atlas) but lacking a vertebral body. The second cervical vertebra of mammals, the axis, is characterized by an appendage termed the dens axis, which is probably homologous to the body of the atlas (but see Jenkins, 1969, for further discussion). The cells that form the proatlas in early vertebrates are thought to form the apex of the dens axis ("body of proatlas") and structures of the occipital bones adjacent to the foramen magnum in the more evolved mammalian forms.

To produce dominant gain-of-function mutations, the spatial expression pattern of a Hox gene was deregulated in transgenic mice. We previously described craniofacial abnormalities in transgenic mice ectopically expressing the murine homeobox gene Hox-1.1 (Balling et al., 1989). Further pursuit of this experimental approach has revealed variations of the cervical vertebral column in severely affected animals, which are consistent with a developmental control function of the Hox-1.1 gene.

\section{Results}

An expression vector $(\mathrm{g} \beta)$ consisting of the transcribed region of the murine homeobox gene Hox-1.1 linked to the chicken $\beta$-actin promoter was microinjected into fertilized oocytes, and transgenic mice were generated as described previously (Balling et al., 1989). Since postnatal lethality due to expression of this transgene had been shown in many independent cases, the litters were delivered on day 19 by cesarian sections. Open eyelids, abnormal external ears, and cleft palate are observed in about $20 \%$ of the transgenic mice. The severity of the phenotype ranges from a small opening of the eyelids to large open- 


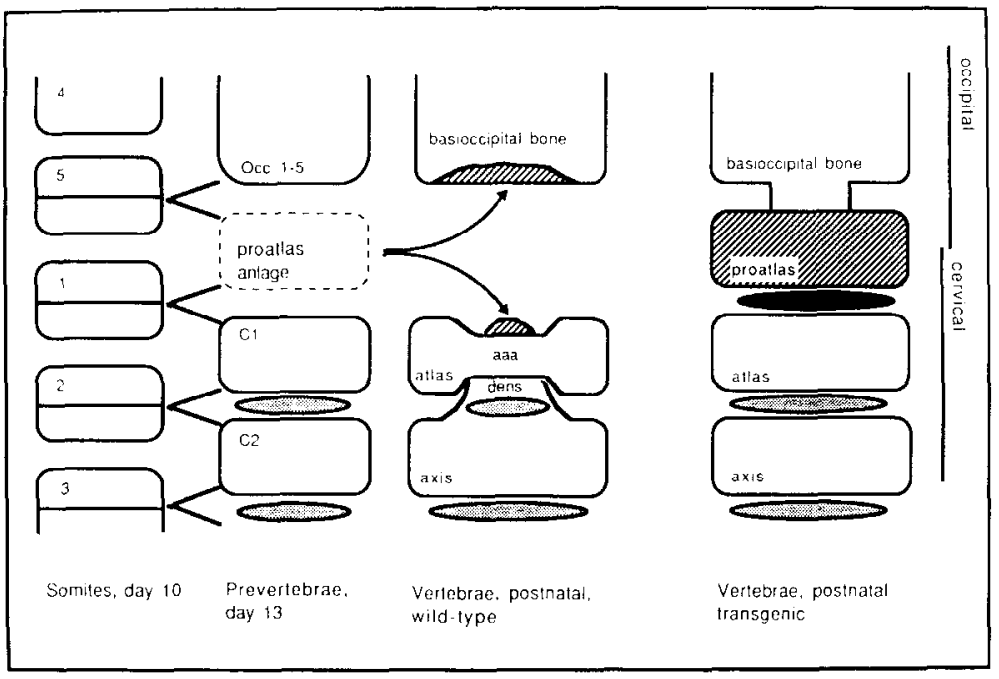

Figure 1. Development of Cervical Somites into Vertebrae

The last occipital somites ( 4 and 5 ) and the first cervical somites $(1,2$, and 3$)$ are depicted with their anterior and posterior halves. They develop into the precursors of the occipital bones (Occ 1-5), the proatlas anlage, and cervical prevertebrae $\left(\mathrm{C}_{1}\right.$ and $\left.\mathrm{C}_{2}\right)$ as indicaled. The vertebral body of $\mathrm{C} 1$ fuses with $\mathrm{C} 2$, resulting in the typical axis with the dens axis. Therefore, the neonatal atlas does not contain a vertebral body. Unique to the atlas is the anterior arch (aaa), which is only a transient embryonic structure for the other vertebrae. Also, the proatlas anlage is transient: these cells fuse to the occipital bones or form the tip of the dens (hatched). Intervertebral discs are shown as ellipsoids (stippled), and the additional disc in the scheme for the transgenic animals is shown in black. A further explanation of the transgeric phenotype is given in the text.

ings with apparently missing tissue around the eyes, from nonfused pinnae to severe malformations of the external ear, and from abnormal rugae patterns on closed palates to complete absence of the secondary palate. We attribute this variable penetrance to influences of the integration site on the transgene, possibly due to the relatively small (340 bp) promoter fragment used for the construction of the vector.

Severely affected animals were studied in more detail using whole-mount alizarin red staining for calcified, bony structures, alcian blue for staining cartilage, and sagittal sectioning of paraffin-embedded newborns. As pointed out before, no specific defects in visceral organs could be detected. We reproducibly observed malformations of the upper cervical column and of occipital bones, which will be described in detail below.

\section{Atlas and Axis}

Evaluation of the complete vertebral column reveals that all except the two most rostrally located vertebrae (atlas and axis) were normal. Figure 2 shows a cranial view of the macroscopic structure of an atlas from a transgenic newborn stained with alizarin blue. A variation from the normally oval shape is visible; the processi transversi are abnormal. The major difference, compared with a normal atlas, is the presence of a vertebral body fused to the anterior arch of the atlas. This tight connection is further demonstrated in midsagittal sections of a different transgenic individual in Figure 3A, while the independence of the anterior arch from the axis is shown in the wild-type control animal (Figure 3B)

The existence of an atlantal vertebral body also has consequences for the formation of the axis. A dens axis in the typical form, consisting of body (atlas derived) and apex (proatlas derived), is not present (Figure 3). The intervertebral disc between axis and atlas body, which separates the bodies of axis and dens in normal mice, separates two complete vertebrae in the transgenic animals. The axis has assumed characteristics of the next caudally positioned vertebra, $\mathrm{C} 3$.
The abnormally developed atlas in newborn transgenic animals resembles a structure transiently present during 'normal embryogenesis (Figure 1). Around day 13 of gestation the cartilaginous prevertebral atlas still contains a body, the body of the dens, which only later becomes part of the axis. The second unique feature of a wild-type atlas, the anterior arch, is normally free from the axis, thus allowing the rotational movement of the head-atlas complex relative to the body. The functional difference in the transgenic animals is that a complex cartilaginous mass consisting of axis, disc, body of atlas, and anterior arch of atlas blocks rotational movements. Judging from human orthopedics, such a fixation might be the cause for severe problems, but would not be expected to be lethal.

In sum, the sophisticated atlantoaxial joint typical of mammals is modified in the transgenic mice to a more primitive configuration. In retaining one vertebral body the atlas as well as the axis has achieved a structure characteristic of more posterior vertebrae.

\section{Proatlas and Occipital Bones}

Figure 3 depicts further major deviations from the vertebral column of a wild-type mouse. While normally the apex dens axis is the most anterior part of the vertebral column, transgenic animals possess additional structures at the craniocervical transition, by which the skull becomes fused to the vertebral column.

An additional intervertebral disc is apparent rostral to the vertebral body of the atlas (Figure 3 ). This disc is macroscopically visible after the anterior arch is mechanically bent (Figure 4) and stains with alcian blue in the same pale-blue color observed for more caudal discs. Also, the fine structure of this intervertebral disc, as illustrated in Figure 3, resembles more caudally located discs. The ventral compressions of all three discs visible in Figure 3 are secondary effects of the malformed upper vertebral column, which also explains the atypically bent neck posture of the animals. An additional intervertebral disc at this position has not been previously described to our knowl- 

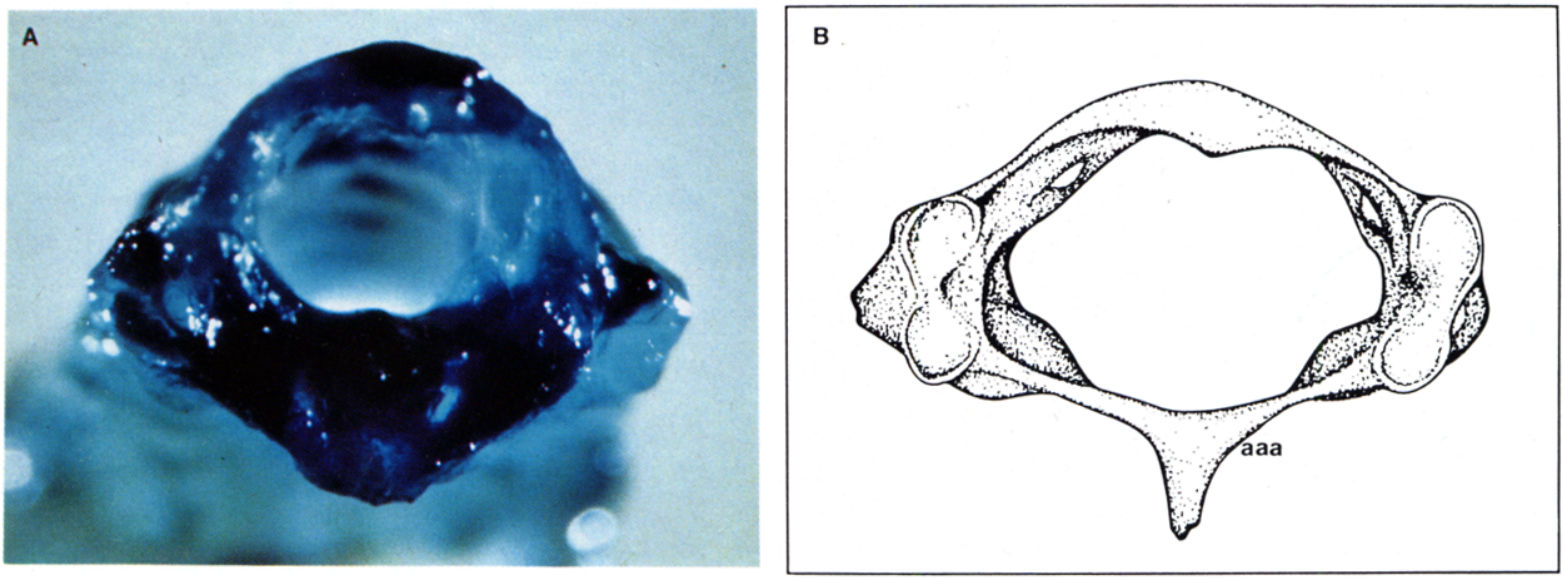

Figure 2. The Vertebral Body of the Atlas from a Transgenic Newborn

(A) Alcian blue staining of a transgenic atlas, cranial view.

(B) Murine wild-type atlas, cranial view (redrawn after Grüneberg [1950] and Leissing [1987]). Note the absence of an atlantal body. The anterior arch of the atlas (arcus anterior atlantis) is marked (aaa).

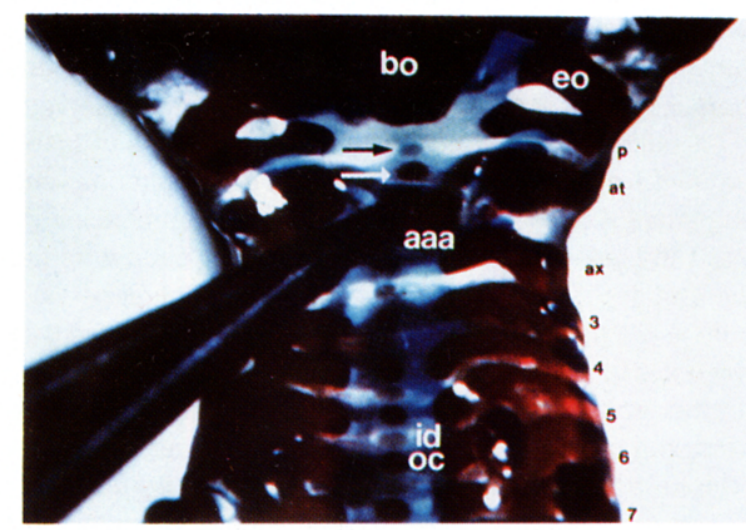

Figure 4. An Additional Intervertebral Disc in Transgenic Mice

Alizarin red/alcian blue staining of a transgenic newborn, ventral view. The anterior arch of the atlas (aaa) has been bent mechanically to allow the view on the ossification center of the atlas (white arrow) and the intervertebral disc (black arrow) between atlas (at) and proatlas ( $p$ ) The photograph was deliberately underexposed to highlight the intervertebral disc. The basioccipital bone (bo), exooccipital bone (eo), axis (ax), cervical vertebrae ( 37 ), ossification center of the sixth cervica vertebra $(O c)$, and the intervertebral disc (id) between vertebrae 5 and 6 are marked.
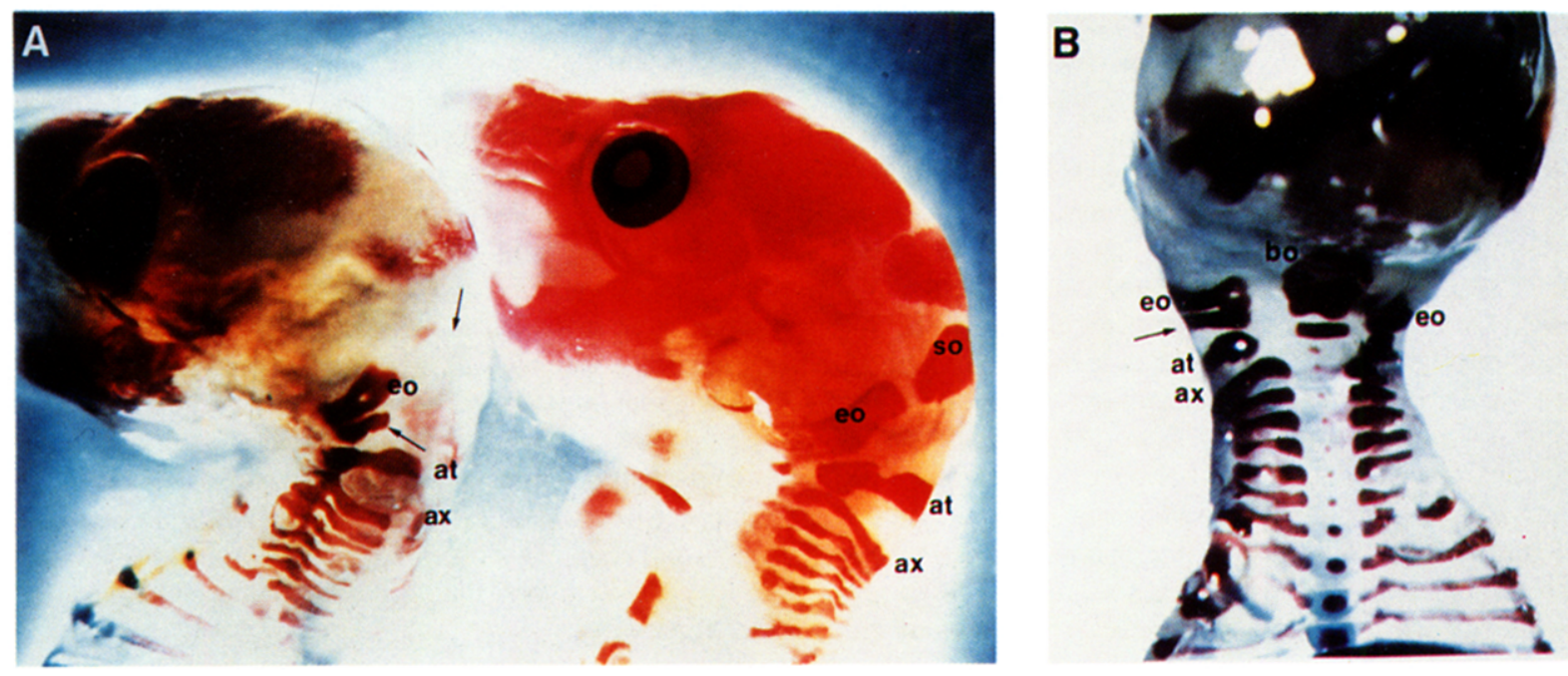

Figure 5. Lateral Ossifications and Missing of the Supraoccipital Bone in Transgenic Animals

(A) Alizarin red stainings of newborn transgenic (left) and wild-type (right) littermates from a dorsolateral view. Exooccipital bone (eo), supraoccipital bone (so), basioccipital bone (bo), atlas (at), and axis (ax) are marked. The arrows point out the additional lateral ossification and the missing supraoccipital bone in the transgenic animal

(B) Ventral view of the transgenic animal. 

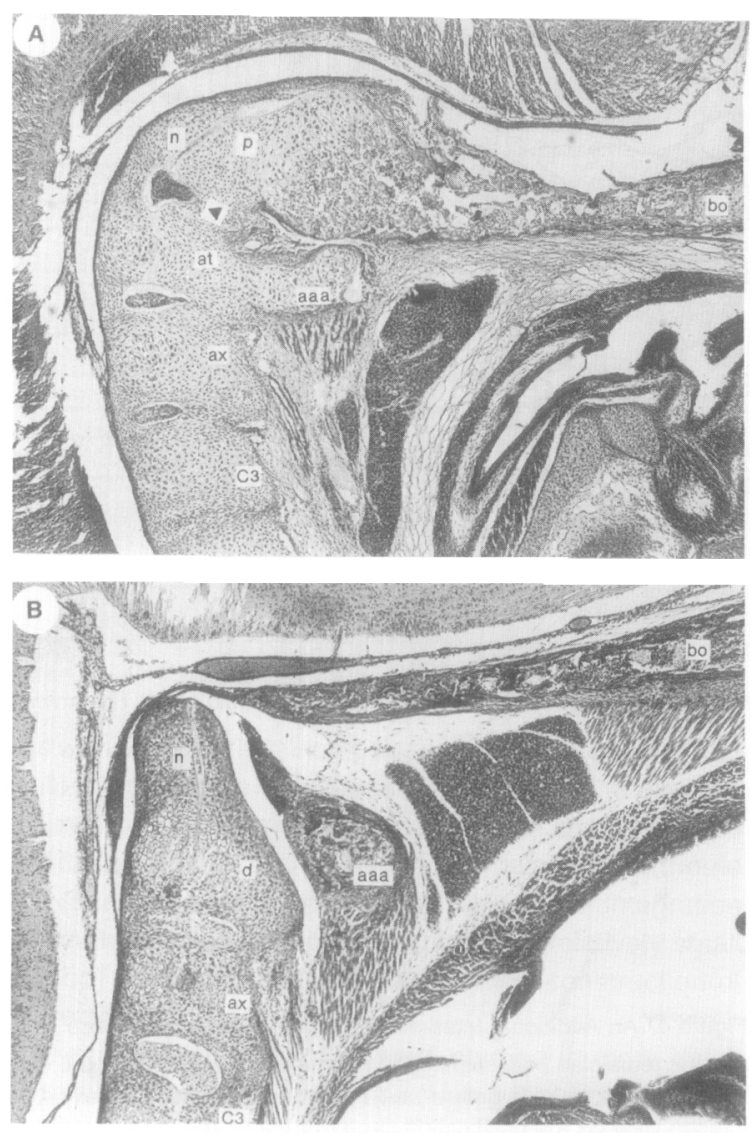

Figure 3. Upper Cervical Vertebral Column

Midsagittal sections are showing the ventral part of the vertebral column. Anterior arch of atlas (aaa), vertebral bodies of proatlas (p), atlas (at), axis (ax), and the third cervical vertebra (C3), notochord (n). and basioccipital bone (bo) are marked. The arrowhead points out the additional intervertebral disc. (A) Transgenic newborn. (B) Wild-type newborn.

edge. As the term "intervertebral" already implies, another vertebra would be expected adjacent to it.

Indeed, we detect in midsagittal sections a rostral vertebral body with a fine structure similar to more caudal bodies (Figure 3 ). The position of this structure indicates that it may be part of a proatlas (see Introduction and Figure 1). On the depicted section the notochord can be followed through the body of the atlas to the proatlas body. The typical widening of the notochord shortly before it ends in the apex dens axis (Theiler, 1988) also occurs in the transgenic proatlas. Here, however, the notochord has reached a more rostral level than normal, owing to the additional vertebral body. The latter also enforces a severe bending of the notochord. In sections or alizarin clearings, we have never observed an ossification center of the proatlas body, nor have we observed ossification of the apex dens axis in wild-type newborn mice. Notably, ossification of the apex dens axis (ossiculum terminale) in humans only occurs a few years after birth (Hayek, 1924; von Torklus and Gehle, 1975).

The proatlas is fused ventrally to the basioccipital bone, which is identified as an ossified structure in Figure 3. Vertebral cells can also be observed rostral to the anterior arch of the atlas, ventral from the proatlas between the occipital bone. They might represent the hypochordal bar of the proatlas, indicating that an intrasegmental fusion between body and anterior arch occurred, very similar to that seen in the adjacent atlantal segment. However, owing to the severe malformations in this area, such an interpretation remains tentative.

Lateral structures at the border between head and neck are more difficult to interpret in histologic sections. They are apparent as lateral lamina sweeping to the vertebral spine dorsally (Figures $6 \mathrm{~A}$ and $6 \mathrm{~B}$ ) and become more clear after whole-mount bone staining. Direct comparisons between littermates reveal additional lateral bony structures (Figures 4 and 5). In wild-type littermates the lateral ossifications of the atlas are adjacent to the ossification of the exooccipital bones. In transgenic animals an additional lateral ossification is observed between these two structures (Figures 4, 5, and 6). We found unilateral and bilateral structures, fused ventrally or dorsally to the exooccipital bone. We interpret them as parts of the proatlantal neural arch. No dorsal ossification equivalent to the dorsal centers seen in all other vertebrae was observed.

As outlined above, the most rostral vertebra is fused two-fold, ventrally and laterally, to the occipital bone. The schematic representation of vertebral development in Figure 1 indicates that cells derived from the caudal half of the last occipital and the cranial half of the first cervical somite will normally not form a vertebra, but will fuse with the occipital bones and the body of dens. We assume that in the transgenic mice these sclerotomal cells have formed a proatlas, but that the program directing the fusion to the basioccipital bone was still carried out.

The basioccipital bone itself is strikingly malformed (Figures 6B and 6C), a syndrome referred to as occipital dysplasia in human orthopedics (Schmidt and Fischer, 1960). Rather than a straight, ossified structure pointing away from the vertebral column in a wide angle, the basioccipital bone was found severely bent or was irregularly shaped in transgenic animals. Owing to the absence of a secondary palate and a fusion of the basioccipital bone to the vertebral column, the anatomy of the head is unusual. Therefore, it remains unclear if the syndrome represents a secondary effect or a primary effect on the occipital somites.

A feature of at least three transgenic animals is the complete absence of an ossified supraoccipital bone. General retardation can not be responsible since other structures like basi- and exooccipital bones are well ossified. Establishing a correlation between the manifestation of a proatlas and absence of a supraoccipital bone and vice versa might help to resolve the developmental fate of the occipital somites.

In sum, the additional intervertebral disc, the fine structure of a vertebral body, the presence of the notochord, and the lateral ossifications are all indicative of a vertebra rostral to the atlas in transgenic animals. Based on the evolutionary and embryological considerations outlined above, we consider the described vertebral structure as 


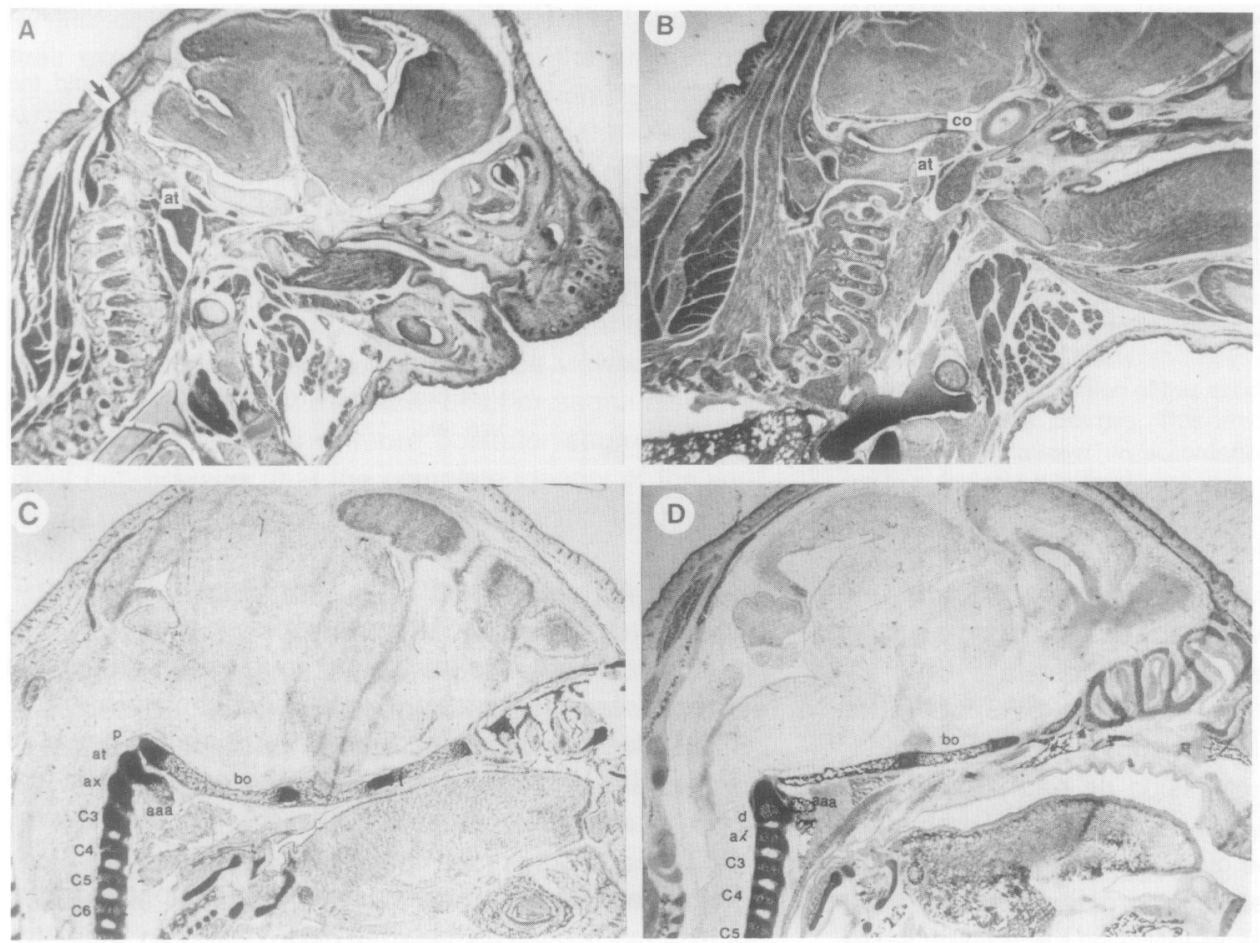

Figure 6. Lateral Ossifications and Occipital Dysplasia in Transgenic Animals.

( $A$ and $B$ ) Parasagittal paraffin sections of a transgenic (A) and a wild-type (B) newborn. Note the lateral ossified structure (arrow) swaying dorsally between occipital bone and atlas (at) in (A), while in the wild-type animal a smooth junction between condyle (co) and atlas (at) is apparent. (C and D) Midsagittal cryosections show the drastic differences of the basioccipital bones (bo). Atlas (at) and axis (ax) are marked, and the posterior cervical vertebrae are numbered (C3-C7). (C) Transgenic newborn. Note the severely bent basioccipital bone, the fusion of the anterior arch of the atlas (aaa) to an atlantal vertebral body, the absence of a dens axis, and the indication of a proatlas vertebral body ( $p$ ). A secondary palate is absent. (D) Wild-type newborn. The dens axis (d) is marked.

the manifestation of a proatlas. The proatlantal body segment, which normally is recognizable only during embryogenesis, is established in the newborn transgenic animal and bears characteristics of the more posterior vertebrae.

\section{Discussion}

\section{Vertebral Phenotypes}

Numerous mouse mutants with vertebral malformations have been studied in great detail (Grüneberg, 1960; Theiler, 1988). They may be classified into disorders of the notochord, of segmentation, or of sclerotome differentiation. Among the diverse phenotypes of the mutants are cervical ribs, fusions of ribs or vertebrae, notched or split vertebrae, dyssymphysis of vertebral arches, and lack of vertebral processes. Only one of these mutants, droopyear, partly shows the specific phenotype we describe in this report (Curry, 1959). Part of the pleiotropic droopy-ear phenotype is that the "tip of dens is incorporated into the basioccipital .... and the dens has reverted to its original role as body of atlas" (Grüneberg, 1960). In addition, these mice have a widespread affliction of the whole skeleton, including skull anomalies causing low-set ears.

The vertebral disorders of undulated mice are of particular interest, since this mutant represents one of the rare cases where the molecular defect of a developmental mutation has been identified (Balling et al., 1988). The undulated mutation colocalizes with the $P a x-1$ gene on chromosome 2. Pax-1 contains a paired box, a sequence homologous to a conserved motif in Drosophila genes, and is expressed in intervertebral discs during embryogenesis (Deutsch et al., 1988). In undulated mice a point mutation changes the amino acid sequence of the Pax-1 protein in the paired domain. undulated mice and also the allelic mutant undulated-extensive show, in addition to malformations in the lumbar region, specific variations in the cervical vertebrae, mainly atlas-axis fusions (Grüneberg, 1950; R. B., unpublished data). It is striking that mutations of both the paired-box gene ( $P a x-1)$ and the homeobox gene (Hox-1.1) cause variations of the upper cervical vertebrae, although not necessarily of the same type. By analogy to developmental control genes in Drosophila, one might predict an involvement of both in a hierachy of regulatory functions.

Can other genes of such a postulated hierarchy of events be identified? Interactions between control genes are one mechanistic example. Thus on the basis of its expression pattern, Hox-1.5 is a candidate, since it is expressed in the most rostral prevertebrae of normal mice (Gaunt, 1988). It is, however, also possible that the ectopic gene product competes for regulatory regions of target genes assumed to be more downstream in the hierarchy. 


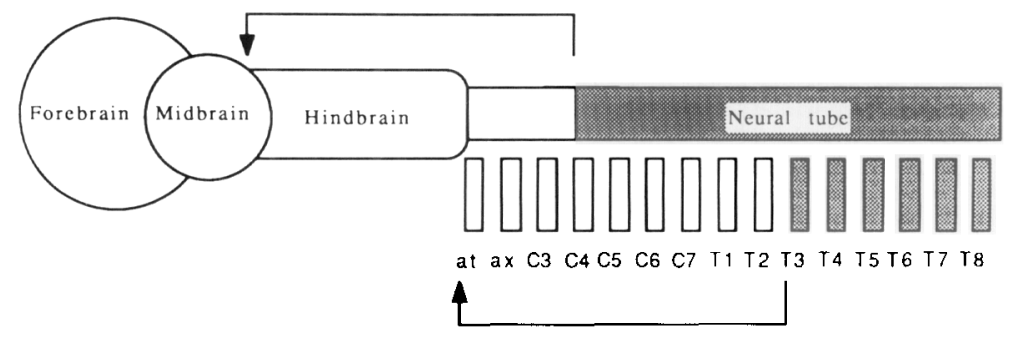

Figure 7. Anterior Expression Boundaries and Sites of Malformations

The anterior neuroectoderm (CNS) and the prevertebrae as metameric units are schematically represented. The expression domains of Hox-1.1 (Mahon et al., 1988) are shown as stippled areas in the CNS (up to the four th cervical ganglion) and in the prevertebrae (up to T3). The arrowheads point out on the anteroposterior axis the sites of malformations occurring in transgenic mice: at the level of the rostral hindbrain (first visceral arch) and at the craniovertebral transition (at). For further explanation see text.

Either repression or activation by the Hox- 1.1 transgene can be envisaged. The availability of a transgenic system may allow these questions to be addressed.

In our previous paper (Balling et al., 1989), the open eye/abnormal ear/cleft palate phenotype was attributed to an effect of transgene expression on neural crest cells originating from the level of the first branchial arch at the time of migration from the closing neural tube (day 8; Le Douarin, 198'). The vertebral syndrome described in this report clearly results from an effect on the somitic mesoderm. It traces back to the early postgastrulation phase, day 8-10 of gestation, when the rostral somites start to differentiate into sclerotome and dermomyotome (Verbout, 1985). Thus it appears that the ubiquitous Hox-1.1 expression interferes with ectoderm as well as mesoderm differentiation at a similar stage of embryogenesis, a stage when the endogenous Hox-1.1 gene is transcribed. As a consequence of extending the anterior border of Hox-1.1 expression, which is precisely demarcated in the neuroectoderm and the somitic mesoderm of wild-type animals (Mahon et al., 1988), malformations of anterior derivatives of both germ layers arise (Figure 7). The distances from the anterior borders of normal expression to the regions of abnormality are the same in ectodermal structures (fourth cervical ganglion to rostral hindbrain) and mesodermal structures (third thoracic prevertebra to first cervical vertebra).

While the craniofacial abnormalities represent disruptive alterations of ectoderm derivatives, the manifestation of a proatlas and the formation of an atlantal vertebral body imply the generation of new mesodermal structures (Garcia-Bellido, 1977). Variations of the vertebral column have fascinated anatomists, biologists, and physicians for a long time. A long list of described phenotypes includes vertebral fusions, extra vertebrae, and changes from cervical vertebrae into thoracic, thoracic into lumbar, or vice versa (Bateson, 1894; Schmidt and Fischer, 1960; von Torklus and Gehle, 1975). Particularly relevant for this discussion are reports of "occipital vertebrae" and other bony structures at the foramen magnum, which often have been interpreted as parts of a proatlas (Gladstone et al., 1915; von Torklus and Gehle, 1975). We found one report (Hunter, 1923) of a human atlas with a vertebral body resembling the transgenic atlas shown in Figure 2 and the atlas of droopy-ear mice (Grüneberg, 1960).

\section{Hox-1.1: A Developmental Control Gene}

An important question for understanding the function of murine homeobox genes is whether they are competent to act as regulatory master genes during embryogenesis. Such a function can be attributed to homeotic genes of Drosophila, which have the ability to select a genetic program for a partial or entire segment (Gehring and Hiromi, 1986).

Homeotic varlations of Urosophila do not occur at random. Even bizarre phenotypes clearly follow evolutionary channels (Raff and Kaufman, 1983). For example, leg-like structures on a head segment, like those found in Antennapedia (Antp) or Proboscipedia, are a feature of myriapod-like precursors of insects. A four-winged insect (Pterygota) is considered evolutionarily older than a two-winged form like Drosophila (Diptera). Reversions to previous evolutionary states can also be recognized in the transgenic mice: continuity of nasal and oral cavity is common in birds and reptiles, an atlantal vertebral body is present in birds, reptiles, and monotremes, and manifestation of the proatlas is a feature of extinct dinosaurs and certain reptiles (Evans, 1939; Hayek, 1924; Jenkins, 1969; Starck, 1979). Thus, as observed in the homeotic insect mutants, manipulating homeobox gene expression in mice has led to atavistic changes.

According to the model of Lewis (1978), homeotic genes specify segment diversity by defining the identity of a segment through the combinatorial action of a spectrum of genes specific for a particular segment. Lewis considered the second mesothoracic segment as a ground state, which is modified by the additional expression or repression of homeotic genes. As predicted by his model, dominant mutations lead to the transformation of a body part to a more posterior characteristic, while recessive mutations result in anterior transformations. Although some additions had to be made recently (Akam et al., 1988; Pfeifer et al., 1987), the general principle has been verified as the basic concept of Drosphila development.

Supporting evidence comes from the analysis of transgenic flies carrying heat shock-controlled homeotic genes, which have recently been generated in a number of laboratories (Gibson and Gehring, 1988; Schneuwly et al., 1987; Kuziora and McGinnis, 1988, 1989; Hogness et al., personal communication). These studies demonstrated that ectopically expressed homeobox transgenes can al- 
ter the identity of body regions to an identity typical for their expression domains. For example, the Antp transgene transforms the four parasegments anterior to the Antp expression domain (parasegment 4 ) to the identity of parasegment 4 , while posterior segments are not affected (Gibson and Gehring, 1988)

The predictions of the Lewis model may also be applied to the vertebral phenotype of the transgenic mice. The axis without a dens, the atlas with a vertebral body, and the manifestation of the proatlas can be viewed as posterior transformations: the more anterior segments have achieved the likeness of more posterior segments. We point out, however, that the complete identity of the vertebrae was not changed, but only the specific features described.

Reverse genetics is most often the only possible approach to study the function of candidate control genes of vertebrates. The generation of a gain-of-function Hox-1.1 mutant is described in this report; first steps toward a lossof-function Hox-1.1 mutant are described elsewhere (Zimmer and Gruss, 1989). Wright et al. (1989) were the first to report a loss-of-function phenotype for a homeobox gene in vertebrates. They injected an anti-Hox-protein antibody into Xenopus oocytes, and thus inactivated the Xenopus XIHbox 1 (HoX-3.3) gene product in the developing tadpole (Wright et al., 1989). The XIHbox 1 gene is normally expressed in a defined region of the anterior spinal cord. The consequence of the manipulation was an anterior transformation: the XIHbox expression domain was transformed into hindbrain, i.e., into the next most anterior structure. Thus not only gain-, but also loss-of-function mutations in ver tebrates follow the predictions of Lewis for Drosophila homeotic genes. The validity of one model for both Drosophila and vertebrate genes would suggest a similar basic function in morphogenesis, such as the establishment of positional information and identity along the anteroposterior axis (De Robertis et al., 1989).

The phenotypic variations of the transgenic mice described in this paper are caused by the deregulated expression of a single homeobox gene. The Hox-1.1 transgene functions as a developmental control gene by switching on a different genetic program or, alternatively, interfering with the programs specifying the upper vertebral column. The results of this reprogramming are not random or disruptive, but can lead to structures representative of earlier ontogenetic or phylogenetic stages. Several aspects of the variations occurring after ectopic expression of the Hox-1.1 transgene are consistent with a homeotic function of this vertebrate homeobox gene.

\section{Experimental Procedures}

Transgenic animals were generated as described previously (Balling et al., 1989). Some litters were dissected a day before birth (day 19 of gestation) in order to circumvent the postnatal lethality. Transgenic mice evident by phenotype were dissected for RNA analysis, leaving the skeleton intact for further analysis. These were processed directly for the clearing technique or were fixed in Bouin's solution and embedded in paraffin

For bone and cartilage staining, animals were skinned and visceral organs removed. After fixation in $99 \%$ ethanol and acetone, specimens were stained in alizarin red $S(0.015 \%)$, alcian blue $8 G X$
$(0,005 \%)$, or a combination of both in $70 \%$ ethanol for 3 days. Clearing was achieved in $20 \%$ glycerol, $1 \% \mathrm{KOH}$ after several weeks.

Paraffin sections $(8-10 \mu \mathrm{m})$ were routinely stained in hematoxylin and eosin, cryosections $(8 \mu \mathrm{m})$ in thionin

\section{Acknowledgments}

We thank Carmen Kropp and Merve Olowson for excellent technical assistance and Ralf Altschäffel for photography. The advice of G. Mutter (Boston), G. Morriss-Kay (Oxford), B. Christ (Bochum), and G. Dressler (Göttingen) is highly appreciated. This work was supported by the Max Planck Society.

The costs of publication of this article were defrayed in part by the payment of page charges. This article must therefore be hereby marked "advertisement" in accordance with 18 U.S.C. Section 1734 solely to indicate this fact

Received November 6, 1989; revised January 15, 1990.

\section{References}

Akam, M., Dawson, 1., and Tear, G. (1988). Homeotic genes and the control of segment diversity. Development 104 (Suppl.), 123-133.

Balling, R., Deutsch, U., and Gruss, P. (1988). undulated, a mutation affecting the development of the mouse skeleton, has a point mutation in the paired box of Pax 1. Cell 55, 531-535.

Balling, R., Mutter, G., Gruss, P., and Kessel, M. (1989). Craniofacial abnormalities induced by ectopic expression of the homeobox gene Hox-1.1 in transgenic mice. Cell 58, 337-347.

Bateson, W. (1894). Materials for the Study of Variation (London: Macmillan).

Baur, R. (1969). Zum Problem der Neugliederung der Wirbelsäule Acta Anat. 72, 321-356

Curry, G. A. (1959). Genetical and developmental studies on droopyear mice. J. Embryol. Exp. Morphol. 7, 39-65.

Dawes, B. (1930). The development of the vertebral column in mammals, as illustrated by its development in Mus musculus. Phil. Trans. Roy. Soc. (Lond.) B 218, 155-170.

De Robertis, E. M., Oliver, G., and Wright, C. V. E. (1989). Determination of axial polarity in the vertebrate embryo: homeodomain proteins and homeogenetic induction. Cell 57, 189-191.

Deutsch, U., Dressler, G. R., and Gruss, P. (1988). Pax 1, a member of the paired box homologous murine gene family, is expressed in segmented structures during development. Cell 53, 617-625.

Evans, F. G. (1939). The morphology and functional evolution of the atlas-axis complex from fish to mammals. Ann. NY Acad. Sci. 39, 29-104

Featherstone, M., Baron, A., Gaunt, S. J., Mattei, M.-G., and Duboule, D. (1988). Hox-5.7 defines a homeobox-containing gene locus on mouse chromosome 2. Proc. Natl. Acad. Sci. USA 85, 4760-4764.

Garcia-Bellido, A. (1977). Homoeotic and atavic mutations in insects. Am. Zool. 17, 613-629.

Gaunt, S. J. (1988). Mouse homeobox gene transcripts occupy different but overlapping domains in embryonic germ layers and organs: a comparison of Hox-3.1 and Hox-1.5. Development 103, 135-144.

Gibson, G., and Gehring, W. (1988). Head and thuracic transformations caused by ectopic expression of Antennapedia during Drosophila development. Development 102, 657-675.

Grüneberg, H. (1950). Genetical studies on the skeleton of the mouse. II. Undulated and its modifiers. J. Genet. 50, 142-173.

Gruneberg, H. (1952). The Genetics of the Mouse (The Hague: Nijnoff)

Grüneberg, H. (1960). The Pathology of Development (Oxford: Blackwell Scientific Publications).

Hayek, H. (1922). Über den Proatlas und über die Entwicklung der Kopfgelenke beim Menschen und bei einigen Säugetieren. Sitzungsberichte d. Akad. d. Wiss. 31, 25-60.

Hayek, H. (1924). Über das Schicksal des Proatlas und über die Ent- 
wicklung der Kopfgelenke bei Reptilien und Vögeln. Jahrbuch für Morphologie und mikroskopische Anatomie 53, 137-163.

Hayek, H. (1927). Untersuchungen über Epistropheus, Atlas und Hinterhauptsbein. Jahrbuch für Morphologie und mikroskopische Anatomie $58,269-347$.

Hill, R. E., Jones, P. F., Rees, A. R., Sime, C. M., Justice, M. J. Copeland, N. G., Jenkins, N. A., Graham, E., and Davidson, D. R. (1989). A new familiy of mouse homeo box-containing genes: molecular structure, chromosomal location, and developmental expression of Hox-7.1. Genes Dev. 3, 26-37.

Hunter, R. H. (1923). An abnormal atlas. J. Anat. 58, 140-141.

Jenkins, F. A. (1969). The evolution and development of the dens of the mammalian axis. Anat. Rec. 164, 173-184.

Kuziora, M. A., and McGinnis, W. (1988). Autoregulation of a Drosophila homeotic selector gene. Cell 55, 477-485.

Kuziora, M. A., and McGinnis, W. (1989). A homeodomain substitution changes the regulatory specificity of the Deformed protein in Drosophila embryos. Cell 59, 563-571.

Le Douarin, N. (1982). The Neural Crest (Cambridge: Cambridge University Press)

Leissing, C. (1987). Vorkommen und Entwicklung der Halsrippen bei der Labormaus. Ph.D. thesis, Universität Zürich.

Lewis, E. B. (1978). A gene complex controlling segmentation in Drosophila. Nature $276,565-570$

Mahon, K. A., Westphal, H., and Gruss, P. (1988). Expression of homeobox gene Hox-1.1 during mouse embryogenesis. Development 104 (Suppl.), 187-195.

McGinnis, W., Garber, R. L., Wirz, J., Kuroiwa, A., and Gehring, W. J. (1984). A homologous protein coding sequence in Drosophila homeotic genes and its conservation in other metazoans. Cell 37, 403-409.

Mock, B. A., D'Hoostelaere, L. A., Matthai, R., and Huppi, K. (1987). A mouse homeobox gene, Hox-1.5, and the morphological locus, $H d$, map to within $1 \mathrm{cM}$ on chromosome 6. Genetics 116, 607-612

Nüsslein-Volhard, C., and Wieschaus, E. (1980). Mutations affecting segment number and polarity in Drosophila. Nature 287, 795-801.

Ouweneel, W. F. (1976). Developmental genetics of homoeosis. Adv Genet. 18, 179-248.

Pfeifer, M., Karch, F., and Bender, W. (1987). The bithorax complex control of segment identity. Genes Dev. 1, 891-898.

Raff, R. A., and Kaufman, T. C. (1983). Embryos, Genes and Evolution (Now York: Macmillan).

Reiter, A. (1944). Die Frühentwicklung der menschlichen Wirbelsäule. II. Die Entwicklung der Occipitalsegmente und der Halswirbelsäule. Z. Anat. Entwicklungsgeschichte 113, 66-104.

Robert, B., Sassoon, D., Jaco, B., Gehring, W., and Buckingham, M (1989). Hox-7, a mouse homeobox gene with a novel pattern of expression during embryogenesis. EMBO J. 8, 91-100.

Schmidt, H., and Fischer, E. (1960). Die Okzipitale Dysplasie (Stuttgart: G. Thieme Verlag).

Schneuwly, S., Klemenz, R., and Gehring, W. (1987). Redesigning the body plan of Drosophila by ectopic expression of the homeotic gene Antennapedia. Nature 325, 816-818.

Scott, M. P. Tamkun, J.W. and Hartzell, G. W. (1989). The structure and function of the homeodomain. Biochim. Biophys. Acta 989, 25-48

Starck, D. (1979). Vergleichende Anatomie der Wirbeltiere (Berlin Springer-Verlag).

Stern, C. D., and Keynes, R. J. (1986). Cell lineage and the formation and maintenance of half somites. NATO ASI Series $718,147-159$.

Theiler, K. (1988). Vertebral malformations. Adv. Anat. Embryol. Cell Biol. 112, 1-99.

Verbout, A. J. (1985). The development of the vertebral column. Adv Anat. Embryol. Cell Biol. 90,1-120

von Torklus, D., and Gehle, W. (1975). Die Obere Halswirbelsäule (Stuttgart: $G$. Thieme Verlag).

Wright, C. V. E., Cho, K. W. Y., Hardwicke, J., Collins, R. H., and De Robertis, E. M. (1989). Interference with function of a homeobox gene in Xenopus embryos produces malformations of the anterior spina cord. Cell 59, 81-93

Zimmer, A., and Gruss, P. (1989). Production of chimaeric mice containig embryonic stem cells carrying a homeobox Hox-1.1 allele mutated by homologous recombination. Nature $338,150-153$

\section{Note Added in Proof}

We have recently obtained a transgenic mouse, born with wide open eyes, one severely malformed and one nonfused ear, and cleft secondary palate. The mouse, however, did not show the phenotype of the upper cervical vertebrae as described above. At the level of the seventh cervical vertebra, an additional rib was detected, resulting in a total of 14 ribs, in contrast to 13 observed in control animals. This finding represents a different kind of posterior transformation, changing a cervical to a thoracical identity. It further demonstrates the homeotic capacity of the Hox-1.1 gene. 